\title{
Fitz-hugh-curtis syndrome: A rare disease in the differential diagnosis of acute abdomen
}

\author{
(1) Ahmet Burak Çiftci', (1) Sönmez Ocak¹, () Abdullah Taylan² \\ 1 University of Health Sciences Turkey, Samsun Training and Research Hospital, Clinic of General Surgery, Samsun, Turkey \\ 2University of Health Sciences Turkey, Samsun Training and Research Hospital, Clinic of Radiology, Samsun, Turkey
}

\section{Date submitted:}

07.01 .2020

Date accepted:

17.03.2020

Online publication date:

15.09.2020

\section{Corresponding Author:}

Ahmet Burak Çiftci, MD, University of Health Sciences Turkey, Samsun Training and Research Hospital, Clinic of General Surgery, Samsun, Turkey drburakciftci@yahoo.com

\section{ORCID:}

orcid.org/0000-0002-1814-4008

Presented in: This manuscript was presented as electronic poster in 12. National Trauma and Emergency Surgery Congress.

Keywords: Fitz-Hugh-Curtis syndrome, perihepatitis, acute abdominal pain, pelvic inflammatory disease

\begin{abstract}
Fitz-Hugh-Curtis syndrome (FHCS) is defined as peritoneal capsule inflammation of the liver. This disease is thought to be a complication of an ascending genital infection. A young sexually active woman was admitted to our emergency department with an acute right upper quadrant abdominal pain and diagnosed as FHCS via computed tomography examination. She was treated conservatively with adequate antibiotherapy. This syndrome should be kept in mind in the differential diagnosis of acute abdominal pain diseases to avoid unnecessary surgery.
\end{abstract}

\section{Introduction}

Fitz-Hugh-Curtis syndrome (FHCS) is a rare syndrome which is characterized by perihepatic inflammation associated with an ascending genital infection. The diagnosis is difficult and it may be misdiagnosed as it may present like many other disorders like acute cholecystitis, right pyelonephritis, pneumonia and even acute appendicitis (1,2). First in 1930, Curtis described adhesions of the anterior surface of the liver, called "violin-string" adhesions, in patients with coincident residual gonococcal tubal disease (3). Four years later, Fitz-Hugh described clinical characteristics of this syndrome (4). Although it is first reported in the literature in 1930's, the disease is still insufficiently known among general surgeons and thus unnecessary surgery can be made on the clinical ground. So, we aimed to present a young woman who was diagnosed as FHCS and treated medically at our clinic.

\section{Presentation of Case}

A 33-year-old female patient was admitted to the emergency department with a severe right upper and lower abdominal pain 
which was exacerbated by movement, breathing and coughing for the past one week. In her medical history, she had a missed abortus one month ago and she had a treatment for vaginal infection at the same time.

On physical examination, tenderness was found in the right lower and upper abdominal quadrant. Abdominal defense and rebound was also positive. Routine blood tests demonstrated white blood cell count of $8100 / \mathrm{mm}^{3}$ and hemoglobin of 10.8 $\mathrm{gr} / \mathrm{dL}$, and biochemical parameters including liver and renal function tests were also normal. With a preliminary diagnosis of acute abdomen, the abdominal computed tomography (CT) was requested. CT revealed transient hepatic attenuation difference with periportal edema and free fluid in the pelvis, and pelvic fat inflammation is also seen (Figure 1, 2). On CT imaging, the appendix vermiformis, and gall bladder were normal. With these findings, patient was hospitalized for followup and treatment. Next day of admission, a control abdomen ultrasonography (USG) was requested. USG showed normal appendix and gall bladder, but ovaries were enlarged, inflamed and had a cystic appearance. With these findings, preliminary diagnosis was made of FHCS secondary to pelvic inflammatory

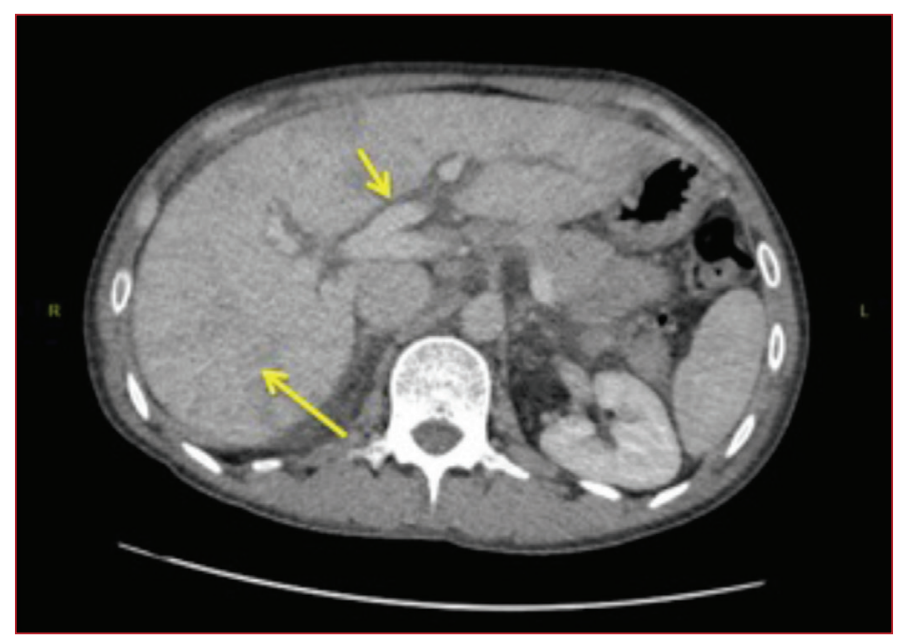

Figure 1. Heterogeneus attenuation pattern in liver parenchyma and periportal halo sign, mild periportal edema

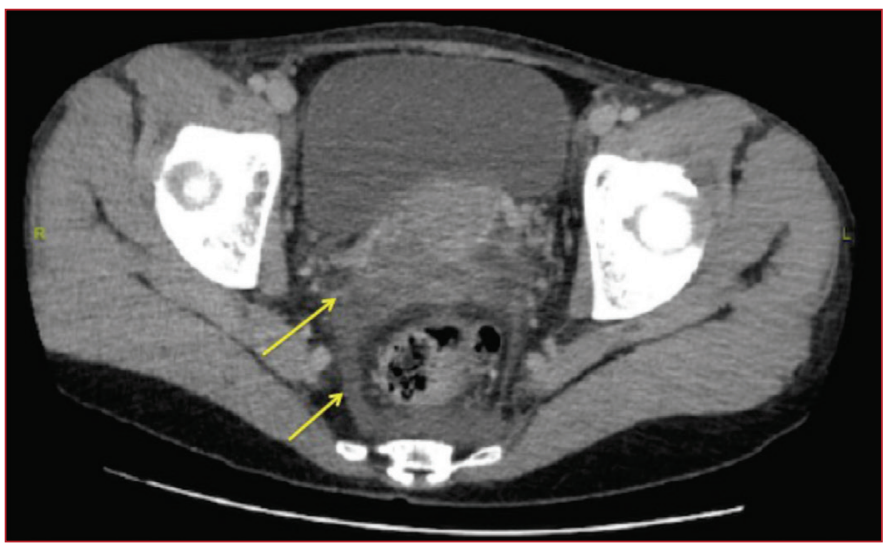

Figure 2. Free fluid in the pouch of Douglas, pelvic fat inflammation and heterogeneous cystic appearance of the ovaries disease (PID). And thus, the medical treatment consisting of ceftriaxone, metronidazole and doxycycline therapy was given, which was derived from the International Union against Sexually Transmitted Infections guideline for PID (5). In the follow-up, the patient recovered well and was discharged on the $7^{\text {th }}$ day of hospitalization.

\section{Discussion}

FHCS is an uncommon condition of the perihepatic capsule inflammation secondary to PID (1). The mechanism of the inflammation is thought to result from the direct intraperitoneal spread of infection towards the perihepatic region from initial pelvic inflammation (6).

Most FHCS patients are sexually active women at childbearing age, who apply to emergency room because of acute pain and tenderness in the right upper abdomen. Because of the physical findings and pain characteristics, it can be difficult to distinguish from acute cholecystitis, sometimes acute appendicitis and the other form of peritonitis $(1,2)$. As in our case, the patient was a young woman at childbearing age and she had a severe acute right upper and lower quadrant abdominal pain and tenderness. So, the first impression of diagnosis was acute cholecystitis or appendicitis. However, CT and USG revealed no gall bladder and appendiceal pathologies. In addition to these imaging findings, liver function tests, bilirubin levels and blood leukocyte counts were also normal. So, despite undergoing an emergency surgery, it was decided to start with a medical treatment. And in our case with the proper treatment, the symptoms were resolved immediateley and no surgical interventions were needed.

In FHCS, the patient's medical history is very important. The prevalence of PID, a history of treatment of sexually transmitted disease or a gynecologic intervention should raise suspicion of this disease (2). Our patient had also a history of a missed abortus and vaginal infection treatment one month ago and she had vaginal discharge as described before.

In the past, the diagnosis was made by showing the perihepatic adhesions via open or laparoscopic surgery and the treatment was made by adhesiotomy. But now, with the development of imaging strategies and antibiotherapy regimens, it can be diagnosed and treated by non-invasive methods $(1,6)$. Contrast enhanced CT is widely accepted as diagnostic method and it provides accurate diagnosis. On CT scan, free fluid in the pouch of Douglas, pelvic fat inflammation, enlarged and inflamed ovaries with a heterogeneous cystic appearance can be reported. In addition to pelvic findings, multiple hypodense periportal halos and decreased parenchymal attenuation of the liver parenchyma and therefore nutmeg liver appearance are also suggestive of the diagnosis of $\operatorname{FHCS}(6,7)$. Our patient had all of these imaging findings. And FHCS was diagnosed rapidly. On the other hand, surgical treatment can be preferred only for 
cases that were unresponsive to antibiotic therapy. Laparoscopic adhesiolysis can be preferred in such cases $(1,2)$.

\section{Conclusion}

FHCS should be kept in mind in the differential diagnosis of acute abdominal pain diseases especially in sexually active women at childbearing age. We hope with this case report that physicians notice this rare syndrome and so unnecessary surgery and diagnostic tools were avoided.

\section{Ethics}

Informed Consent: Consent form was filled out by the participant.

Peer-review: Externally peer-reviewed.

\section{Authorship Contributions}

Surgical and Medical Practices: A.B.Ç., S.O., A.T., Concept: A.B.Ç., S.O., Design: A.B.Ç., S.O., Data Collection or Processing: A.B.Ç., Analysis or Interpretation: A.B.Ç., Literature Search: A.B.Ç., Writing: A.B.Ç., S.O.

Conflict of Interest: No conflict of interest was declared by the authors.
Financial Disclosure: The authors declared that this study received no financial support.

\section{References}

1. Woo SY, Kim JI, Cheung DY, et al. Clinical outcome of FitzHugh-Curtis syndrome mimicking acute biliary disease. World J Gastroenterol. 2008;14:6975-6980.

2. Faré PB, Allio I, Monotti R, Foieni F. Fitz-Hugh-Curtis syndrome: a diagnosis to consider in a woman with right upper quadrant abdominal pain without gallstones. Eur J Case Rep Intern Med. 2017;5:000743.

3. Curtis $\mathrm{AH}$. A cause of adhesions in the right upper quadrant. JAMA. 1930;94:1221-1222.

4. Fitz-Hugh T Jr. Acute gonococcic peritonitis of the right upper quadrant in women. JAMA. 1934;102:2094-2096.

5. Duarte R, Fuhrich D, Ross JDC. A review of antibiotic therapy for pelvic inflammatory disease. Int J Antimicrob Agents. 2015;46:272-277.

6. De Boer JP, Verpalen IM, Gabriels RY, et al. Fitz-HughCurtis syndrome resulting in nutmeg liver on computed tomography. Radiology Case Rep. 2019;14:930-933.

7. Sam JW, Jacobs JE, Birnbaum BA. Spectrum of CT Findings in Acute Pyogenic Pelvic Inflammatory Disease. Radiographics. 2002;22:1327-1334. 